\title{
Biotransformation of Sulfonamide Antibiotics in Activated Sludge: Formation of Pterin- Conjugates Leads to Sustained Risk
}

Stefan Achermann ${ }^{1,2}$, Valeria Bianco ${ }^{1,2}$, Cresten B. Mansfeldt ${ }^{1}$, Bernadette Vogler ${ }^{1}$, Boris A.

Kolvenbach $^{3}$, Philippe F.X. Corvini ${ }^{3,4}$, Kathrin Fenner ${ }^{1,2,5, *}$

${ }^{1}$ Eawag, Swiss Federal Institute of Aquatic Science and Technology, 8600 Dübendorf, Switzerland.

${ }^{2}$ Institute of Biogeochemistry and Pollutant Dynamics, ETH Zürich, 8092 Zürich, Switzerland.

${ }^{3}$ Institute for Ecopreneurship, School of Life Sciences, University of Applied Sciences and Arts

Northwestern Switzerland, 4132 Muttenz, Switzerland. ${ }^{4}$ State Key Laboratory for Pollution Control

and Resource Reuse, School of the Environment, Nanjing University, Nanjing 210093, PR China.

${ }^{5}$ Department of Chemistry, University of Zürich, 8057 Zürich, Switzerland.

*Corresponding author (email: kathrin.fenner@eawag.ch)

\section{Word count:}

Words: 5828

Tables: 2x300 words

Figures: 4x300 words

Total: 7628 words

This document is the accepted manuscript version of the following article: Achermann, S., Bianco, V., Mansfeldt, C. B., Vogler, B., Kolvenbach, B. A., Corvini, P. F. X., \& Fenner, K. (2018). Biotransformation of sulfonamide antibiotics in activated sludge: the formation of pterin-conjugates leads to sustained risk. Environmental Science and Technology. http://doi .org/10.1021/acs . est. 7b06716 


\section{Abstract}

2 The presence of antibiotics in treated wastewater and consequently in surface and ground water

3 resources raises concerns about the formation and spread of antibiotic resistance. Improving removal

4 of antibiotics during wastewater treatment therefore is a prime objective of environmental engineering.

5 Here we obtained a detailed picture of the fate of sulfonamide antibiotics during activated sludge

6 treatment using a combination of analytical methods. We show that pterin-sulfonamide conjugates,

7 which are formed when sulfonamides interact with their target enzyme to inhibit folic acid synthesis,

8 represent a major biotransformation route for sulfonamides in laboratory batch experiments with

9 activated sludge. The same major conjugates were also present in the effluents of nine Swiss wastewater treatment plants. Demonstration of this biotransformation route, which is related to

11 bacterial growth, helps explain seemingly contradictory views on optimal conditions for sulfonamide

12 removal. More importantly, since pterin-sulfonamide conjugates show retained antibiotic activity, our

13 findings suggest that risk from exposure to sulfonamide antibiotics may be less reduced during

14 wastewater treatment than previously assumed. Our results thus further emphasize the inadequacy of

15 focusing on parent compound removal and the importance of investigating biotransformation pathways and removal of bioactivity to properly assess contaminant removal in both engineered and natural systems.

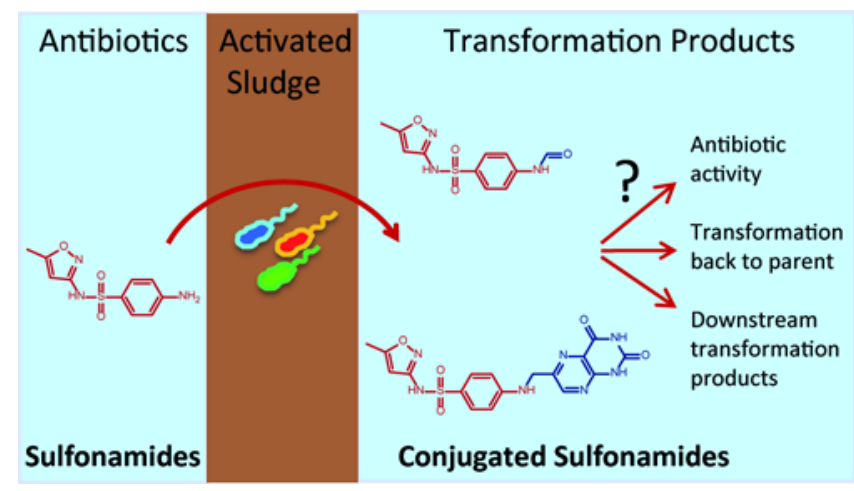




\section{Introduction}

The widespread presence of antibiotics in the environment raises significant concern because their concentrations are high enough to potentially affect sensitive aquatic ecosystems and their ubiquity may support the propagation of antibiotic resistant genes. ${ }^{1-5}$ Sulfonamides, one of the oldest families of antibiotics, are still widely used as human and veterinary drugs. ${ }^{6-8}$ They have been detected in various environmental matrices including soil, wastewater, surface water and ground water. ${ }^{6,-11}$ Particularly for sulfamethoxazole, the sulfonamide antibiotic with the highest reported human consumption, concentrations in the upper $\mathrm{ng} / \mathrm{L}$ to low $\mu \mathrm{g} / \mathrm{L}$ range are frequently detected in municipal wastewater. ${ }^{12-17}$ Biological wastewater treatment plays an important role in reducing the load of chemicals collected by the sewer system prior to discharge to surface waters. ${ }^{18}$ Several studies have investigated the sulfonamide removal capacity of biological wastewater treatment systems, ${ }^{12-16}$ reporting variable degrees of removal ${ }^{19}$ and contradictory results regarding optimal removal conditions. ${ }^{20-25}$

Although removal alone has been repeatedly demonstrated to be insufficient in assessing environmental risk, ${ }^{26,27}$ only limited research has been focused on obtaining a clear picture of the fate of sulfonamides during wastewater treatment. Previous investigations have demonstrated that sorption to activated sludge solids and abiotic processes play a minor role and that the majority of observed removal in activated sludge is linked to biotransformation. ${ }^{21,}{ }^{23}$ However, sulfonamide biotransformation pathways and related transformation product (TP) formation have been little studied. A number of studies focus on the sulfonamide biotransformation capabilities of isolated microbial strains. Several of these studies report the formation of the SMX cleavage product $3 \mathrm{~A} 5 \mathrm{MI},{ }^{28,}$ ${ }^{29}$ or analogous cleavage products for other sulfonamides. ${ }^{30}$ One pure culture study performed with ${ }^{14} \mathrm{C}$-labeled SMX demonstrated partial mineralization to ${ }^{14} \mathrm{CO}_{2} \cdot{ }^{31}$ Another possible biotransformation pathway is related to the original mode of action of sulfonamide antibiotics. The bacteriostatic effect of sulfonamides is based on the competitive inhibition of dihydropteroate synthase (DHPS), a key enzyme involved in intracellular folic acid synthesis. In studies with Escherichia coli and, more recently, in different algal species, sulfonamides were shown to act as alternate substrates for the 
DHPS enzyme, leading to the formation of pterin-sulfonamide conjugates. ${ }^{32-35}$ Whereas these studies show that certain microorganisms are able to degrade sulfonamides or even use them as carbon source, pure culture results generally cannot be extrapolated to mixed communities. ${ }^{36}$

Limited research has focused on the elucidation of sulfonamide biotransformation in complex activated sludge communities. In a recent study with ${ }^{14} \mathrm{C}$ labeled SMX, the parent compound was degraded under aerobic as well as anaerobic conditions, but mineralization rates were below $5 \%$ under all measured conditions, suggesting that the majority of the spiked micropollutants was still present in the form of unidentified transformation products. ${ }^{21}$ In a different study, a number of SMX metabolites were detected including products potentially formed via acetylation, hydroxylation, nitration, deamination or formylation. ${ }^{37}$ However, no attempts were made to prioritize the detected TPs and their relative importance remains unclear. Yet, such knowledge is highly desirable to properly assess risk given the fact that sulfonamide metabolites with modifications at the para-amino position, including those that are formed when sulfonamides interact with their target enzyme to inhibit folic acid synthesis, ${ }^{32-34}$ have been shown to still exhibit antibiotic activity. ${ }^{38,39}$

The objective of this study therefore was to gain a comprehensive view of the transformation pathways and products of sulfonamide antibiotics during wastewater treatment. To this end, we used a combination of two complementary methods, i.e., mass balance analyses with ${ }^{14} \mathrm{C}$-labeled sulfonamides and in-depth transformation product screening with high-resolution mass spectrometry of samples from both laboratory batch experiments with activated sludge and field study samples from wastewater treatment plants. Experiments were performed with five non-radiolabeled sulfonamides in parallel (i.e., sulfamethoxazole, sulfadiazine, sulfamethazine, sulfapyridine and sulfathiazole) to evaluate the generalizability of our findings to the entire family of sulfonamide antibiotics.

\section{Methods}

\section{Batch Experiments}

For the experiments with radiolabeled sulfonamides, ${ }^{14} \mathrm{C}-\mathrm{SMX}$ (aniline $\left[{ }^{14} \mathrm{C}\right]$, Hartmann Analytic) and ${ }^{14} \mathrm{C}-\mathrm{SDZ}$ (2-Pyrimidyl $\left[{ }^{14} \mathrm{C}\right]$-sulfadiazine, Bayer HealthCare) were used. Further details on chemical reference materials are provided in section S1 of the Supporting Information (SI). For all experiments, 
amber glass bottles (100 mL, Schott) were used as batch reactors. To maintain aerobic conditions, caps with two holes were used as previously described. ${ }^{40}$ After sulfonamide addition, the reactors were placed on a circulating shaker table $(160 \mathrm{rpm})$ for the duration of the experiment and samples were collected at different time points as specified below. Batch reactor experiments with SMX and SDZ, with SMZ, SPY and STZ and with pterin-STZ, respectively, were conducted in three separate campaigns. Therefore, fresh activated sludge was collected three times (21.03.2017 (AS1), 12.06.2017 (AS2) and 18.07.2017 (AS3), less than three hours before the experiments were started) from the same aerated nitrifying treatment basin of the same WWTP (ARA Neugut, Dübendorf, Switzerland, details in section $\mathrm{S} 2$ in the SI). During all three experimental campaigns, $\mathrm{pH}$ values remained in the range between 7.87 and 8.28 at all measured time points. Results from measurements of total suspended solids (TSS), $\mathrm{pH}$ and nitrogen species $\left(\mathrm{NH}_{4}{ }^{+}, \mathrm{NO}_{2}{ }^{-}, \mathrm{NO}_{3}{ }^{-}\right)$, and a comprehensive table of all batch experiments including added chemicals, sampling time points and sample volumes is provided in the SI (section S2).

\section{Experiment with Radiolabeled and Non-Labeled SMX and SDZ}

Triplicate biotransformation batch reactors with radiolabeled and non-labeled substances were established in parallel, both for SMX and SDZ. Additionally, abiotic controls with autoclaved (30 min at $125^{\circ} \mathrm{C}$ ) activated sludge and radiolabeled sulfonamides were established in triplicate, and three reactors with only activated sludge added served as non-spiked controls. Biotransformation batch reactors with ${ }^{14} \mathrm{C}$-labeled compounds were spiked (40 $\mu \mathrm{L}$ each, $1.53 \mathrm{kBq} / \mu \mathrm{L}(\mathrm{SMX})$ and $1.02 \mathrm{kBq} / \mu \mathrm{L}$ (SDZ) in methanol), resulting in final initial activities of $61.2 \mathrm{kBq}(\mathrm{SMX})$ and $40.8 \mathrm{kBq}$ (SDZ), respectively, corresponding to initial concentrations of $405 \mu \mathrm{g} / \mathrm{L}$ (SMX) and $115 \mu \mathrm{g} / \mathrm{L}$ (SDZ). Reactors with non-labeled sulfonamides were spiked ( $40 \mu \mathrm{L}$ each, $250 \mathrm{mg} / \mathrm{L}$, in methanol/water 1:3) to achieve initial concentrations of $250 \mu \mathrm{g} / \mathrm{L}$ (SMX and SDZ). To ensure equal starting conditions in terms of carbon availability, the reactors with non-labeled sulfonamides and the non-spiked controls were amended with $30 \mu \mathrm{L}$ and $40 \mu \mathrm{L}$ of methanol, respectively.

Samples were collected over 72 hours. The samples were centrifuged at $21500 \times g$ for 10 minutes at room temperature and the supernatant transferred into amber HPLC vials. For the non-radioactive 
samples, internal standard solution was added (final sample concentration: $24 \mu \mathrm{g} / \mathrm{L}$, details in section S3). Samples were stored at $4{ }^{\circ} \mathrm{C}$ and then analyzed by high-performance liquid chromatography (HPLC) coupled to a high-resolution mass spectrometer (see below for details) within 7 days. For the reactors spiked with radiolabeled sulfonamides, $30 \mu \mathrm{L}$ of supernatant was transferred into a $6 \mathrm{~mL}$ polypropylene vial and mixed with $5 \mathrm{~mL}$ of scintillation cocktail (IrgaSafe Plus, Perkin Elmer) for liquid scintillation counting (LSC). In parallel, $750 \mu \mathrm{L}$ of supernatant were pipetted into amber HPLC vials, stored at $4{ }^{\circ} \mathrm{C}$ and then analyzed by HPLC coupled to a diode array detector (DAD) and an LSC detector within 3 days.

To analyze the radioactivity adsorbed to or incorporated into the biomass, the compressed solid material resulting from centrifugation was obtained after removal of the remaining supernatant. The cell pellet was washed with $\mathrm{NaOH}(1 \mathrm{~mL}, 1 \mathrm{M}$ in water) and the washing liquid was collected after another centrifugation run (15 $\mathrm{min}$ at $21500 \mathrm{rpm})$. The entire washing liquid (1 mL) was mixed with scintillation cocktail (5 mL, Hionic Fluor, Perkin Elmer) and analyzed using LSC. The washed cell pellet was stored at $-20^{\circ} \mathrm{C}$ until analysis in the sample oxidizer as described below. The washing solution contained radioactivity both from ${ }^{14} \mathrm{C}$-SA weakly adsorbed to the microbial cells and from remaining aqueous supernatant that was not removed after centrifugation. Since a major fraction of the radioactivity originated from the remaining aqueous fraction and, as we could show for SMX, the fraction attributed to sorption was not exceeding $4 \%$ at all investigated time points, the measured radioactivity of the washing solution was not considered in the mass balance analysis (details in section S4 in the SI).

\section{Experiment with Non-Labeled SPY, SMZ, STZ and N4-Acetyl-SMX}

Biotransformation experiments with the SAs SMZ, STZ and SPY, and the TP N4-acetyl SMX were run in duplicate. To estimate the degree of sorption and abiotic degradation, SAs were added to duplicate control reactors with autoclaved $\left(121^{\circ} \mathrm{C}\right.$ and $103 \mathrm{kPa}$ for $\left.20 \mathrm{~min}\right)$ activated sludge and activated sludge filtrate, respectively. Non-spiked reactors (duplicate) were run to serve as controls in suspect screening. Samples were collected over $72 \mathrm{~h}$ and processed similarly to the methods described above. For the biotransformation reactors, $25 \mu \mathrm{L}$ of each sulfonamide or N4-acetyl-sulfamethoxazole 
solution (100 mg/L, in methanol/water 1:9) were spiked each into the according batch reactors resulting in a starting concentration in the reactors of $50 \mu \mathrm{g} / \mathrm{L}$. Samples were centrifuged ( $5 \mathrm{~min}$ at $21130 \times g)$, and the supernatant $(750 \mu \mathrm{L})$ was transferred to amber HPLC vials. After the addition of the internal standard solution, samples were stored for $3-7$ days at $4{ }^{\circ} \mathrm{C}$ until HPLC-MS/MS analysis.

\section{Experiment with Pterin-STZ}

A reference standard for pterin-STZ was custom synthesised by SynphaBase, Switzerland. ${ }^{34}$ Six batch reactors were filled with activated sludge of which three were spiked with pterin-STZ ( $25 \mu \mathrm{L}, 100$ $\mathrm{mg} / \mathrm{L}$ pterin-STZ, in methanol/water 1:9) and three served as non-spiked controls. Samples were collected over time and centrifuged $(10 \mathrm{~min}$ at $21130 \times g)$, and the supernatant $(500 \mu \mathrm{L})$ was transferred to amber HPLC vials and stored (for 3-5 days) at $4{ }^{\circ} \mathrm{C}$ until LC-MS/MS analysis.

\section{LC-HRMS/MS Analysis}

All non-radioactive samples were analyzed by reversed-phase HPLC coupled to a high-resolution tandem mass spectrometer (HRMS/MS) (Q Exactive or Q Exactive Plus, Thermo Fisher Scientific). The separation of the analytes was achieved using a C18 silica-based column (Atlantis-T3, particle size $3 \mu \mathrm{m}, 3.0 \times 150 \mathrm{~mm}$, Waters) at $30{ }^{\circ} \mathrm{C}$. Samples from the experiments with non-labeled SPY, SMZ and STZ were measured using an additional guard cartridge (particle size $3 \mu \mathrm{m}, 3.9 \times 20 \mathrm{~mm}$, Waters) leading to higher retention times (RTs). For all samples, $100 \mu \mathrm{L}$ of sample was injected onto the column at a flow rate of $300 \mu \mathrm{L} / \mathrm{min}$. Further analytical details including chromatographic separation and mass spectrometric analysis are provided in section S5 in the SI. For quantification, calibration curves were prepared in nanopure water (Barnstead Nanopure, Thermo Scientific) ranging from $0.2-300 \mu \mathrm{g} / \mathrm{L}$ (SMX and SDZ) and 0.2-75 $\mu \mathrm{g} / \mathrm{L}$ (SPY, SMZ, STZ and TPs). To account for compound losses and interferences during LC-HRMS measurements, internal standards were added to all samples, including the calibration samples (details in sections S3 and S5 in the SI). Quantification was performed using Tracefinder EFS 3.2 (Thermo Scientific) for all parent sulfonamides and for transformation products for which reference standards were available. The lowest calibration standards with a meaningful, detectable peak (reasonable peak shape, a minimum of 4 scans per peak and a 
minimum intensity of 1E04 in Xcalibur Qualbrowser 3.0 (Thermo Scientific)) were regarded as limits of quantification (Table S5.2).

\section{Analysis of Radioactive Residues}

156 Total radioactivity of the samples was assessed using a liquid scintillation counter (Tri-Carb 2800TR,

157 Perkin Elmer). Sample aliquots were mixed with scintillation cocktail prior to the measurements as

158 described above. For analysis of the radioactivity accumulated in the solids, the biomass fractions of

159 the samples were combusted for $1.5 \mathrm{~min}$ in a 307 Perkin Elmer Sample Oxidizer. The resulting ${ }^{14} \mathrm{CO}_{2}$

160 was absorbed by Carbo-Sorb ${ }^{\circledR}$ E (Perkin Elmer) and LSC cocktail (Permafluor ${ }^{\circledR}$ E+, Perkin Elmer)

161 was added. The radioactivity was then assessed by liquid scintillation counting. Radioactive

162 supernatant samples were analyzed using a HPLC 1200 Series (Agilent Technologies) including a

163 diode array detector (DAD) coupled to a subsequent liquid scintillation counter (Ramona Star,

164 Raytest). The DAD signal was recorded at $285 \mathrm{~nm}$ (4 $\mathrm{nm}$ bandwidth), which is close to the maximum

165 of an absorption peak between 250 and $300 \mathrm{~nm}$ in the absorption spectra of both SMX and SDZ. ${ }^{41,42}$

166 An identical column and gradient program and identical eluent mixtures and injection volumes for

167 chromatographic separation as described for the LC-HRMS/MS measurements above were used.

\section{Suspect Transformation Product Screening}

169 For the identification of TPs, a suspect screening was performed using Compound Discoverer 2.1

170 (Thermo Scientific) and Xcalibur Qualbrowser 3.0 (Thermo Scientific). Mass lists for the suspect screening were compiled using in silico prediction with the EAWAG pathway prediction system

172 (EAWAG-PPS, http://eawag-bbd.ethz.ch/predict/). Additionally, previously reported transformation

173 products and mass shifts of typical biotransformation reactions were considered. Details on the

174 compilation of the suspect TP lists and Compound Discoverer settings are further described in section $175 \quad$ S6 in the SI.

176 A suspect TP screening was performed for SMX, SDZ, SMZ, STZ, SPY, N4-acetyl-SMX and pterin177 STZ. For the N4-acetyl-SMX and the pterin-STZ spike experiments, the suspect TP lists of SMX and 178 STZ, respectively, were used. Measurements from replicate batch reactors were grouped in Compound 179 Discoverer. Further analysis and presentation of results was performed based on mean values from 
replicate sample values. Detected features matching suspected TP masses were further assessed: Clear differences in time trends between samples and non-spiked controls were ensured, and isotope patterns were compared with calculated isotope patterns of corresponding suspected molecular formulas. For TPs for which a reference standard was available, retention times of suspected TP and reference could be compared. For TPs for which no reference standards were available, $\mathrm{MS}^{2}$ spectra were compared with library spectra or interpreted manually. In doing so, the $\mathrm{MS}^{2}$ spectra of the TPs were compared with the spectra of the parents or related TPs and with fragments predicted using Mass Frontier 7.0 (HighChem). Based on this procedure, confidence levels were assigned to each of the detected TPs as proposed by Schymanski et al., ranging from level 5 "exact mass", level 4 "unequivocal molecular formula", level 3 "tentative candidate(s)", level 2 "probable structure" to level 1 "confirmed structure" ${ }^{43}$ Details and $\mathrm{MS}^{2}$ spectra are provided in section S7 in the SI.

\section{Wastewater Treatment Plant Samples}

From each of nine Swiss WWTPs, three influent and three effluent samples of the biological treatment step were obtained (1-L aliquots of three consecutive 24-h composite samples; flow-proportional sampling except for WWTP3, in which no SMX was detected in influent or effluent, see results) and combined to $72-\mathrm{h}$ composite samples $(3 \mathrm{~L})$ in our laboratory. The samples were collected during May and August 2013 and stored at $-20{ }^{\circ} \mathrm{C}$ until sample preparation. The samples were enriched by solid phase extraction (SPE) and analyzed by HPLC-MS/MS using a modified protocol based on Moschet $e t$ $a l .{ }^{44}$ (further details in section S8 in the SI). A suspect TP screening was performed with the 5 SMX TPs N4-acetyl-SMX, PtO-SMX, pterin-SMX, Ac-OH-SMX and N4-formyl-SMX using Tracefinder 4.1 (ThermoFisher Scientific) and confidence levels were assigned according to Schymanski et al. ${ }^{43}$ Reasonable peak shapes were ensured by visual inspection and isotope patterns were compared with predicted isotope patterns to confirm molecular formulas. Confidence levels assigned in batch experiments were adopted if at least for two fragments (found in batch experiments or library spectra) their extracted ion chromatograms matched the retention times and peak shapes of the corresponding $\mathrm{MS}^{1}$ extracted chromatograms. To compensate for matrix effects and possible analyte losses during sample preparation and analysis, the detected peak areas were normalized by the peak areas of the 
internal standard (isotope-labeled SMX). Influent SMX concentrations were quantified using the

internal standard method described for the laboratory experiments.

\section{Results}

\section{Biotransformation as Main Removal Mechanism}

Analysis of concentration-time series from batch experiments revealed mean removals of $99.3 \%$ (sulfathiazole, STZ), 88.7\% (sulfapyridine, SPY), 63.8\% (sulfadiazine, SDZ), 63.5\% (sulfamethazine, SMZ) and 58.9\% (sulfamethoxazole, SMX) after 72 hours (from initial concentrations of $405 \mu \mathrm{g} / \mathrm{L}$ (SMX), $115 \mu \mathrm{g} / \mathrm{L}$ (SDZ) and $50 \mu \mathrm{g} / \mathrm{L}$ (SMZ, SPY, STZ)). These removals are comparable to previously reported removals from laboratory studies. ${ }^{24,45}$ Calculation of TSS-normalized pseudo-first order rate constants resulted in values of $0.083 \pm 0.006$ (SMX), $0.093 \pm 0.009$ (SDZ), $0.11 \pm 0.01$ (SMZ), $0.45 \pm 0.01(\mathrm{STZ})$ and $0.20 \pm 0.01(\mathrm{SPY}) \mathrm{L} /\left(\mathrm{g}_{\mathrm{TSs}} \times \mathrm{d}\right)$ and coefficients of determination $\left(\mathrm{r}^{2}\right)$ of at least 0.95 (see details in section S2 in the SI). In the experiments with ${ }^{14} \mathrm{C}$-labeled SMX and SDZ, the sum of the recovered radioactivity in the aqueous and solids fraction accounted for 97.3-105.3\% (SMX) and $97.2-99.0 \%$ (SDZ) of the initially added radioactivity at all time points (Figure 1, section S9 in the Supporting Information (SI)). This indicated that the loss of radioactivity as ${ }^{14} \mathrm{CO}_{2}$ and, therefore, the degree of mineralization must be low, which was consistent with the low fractions of ${ }^{14} \mathrm{CO}_{2}$ recovered from $\mathrm{CO}_{2}$ traps in preliminary experiments and the fact that we did not find any evidence of significant amounts of dissolved ${ }^{14} \mathrm{CO}_{2}$ accumulated in the batch reactors (details in section $\mathrm{S} 10$ in the $\mathrm{SI}$ ).

The fraction of radioactivity recovered from the washed solids (see methods for details on solids washing step) was generally small, but showed an increase over time (72 h) with final maximum values accounting for $6 \%(\mathrm{SMX})$ and 3\% (SDZ) of the initially spiked radioactivity. Experiments with autoclaved activated sludge further confirmed that only small fractions of the spiked sulfonamides (SAs) were sorbed to the activated sludge and that abiotic transformation was negligible (section S11 in the SI). Therefore, the observed declines in parent sulfonamide concentrations can be confidently attributed to biotransformation. Together with the observation that the major part of the radioactivity 


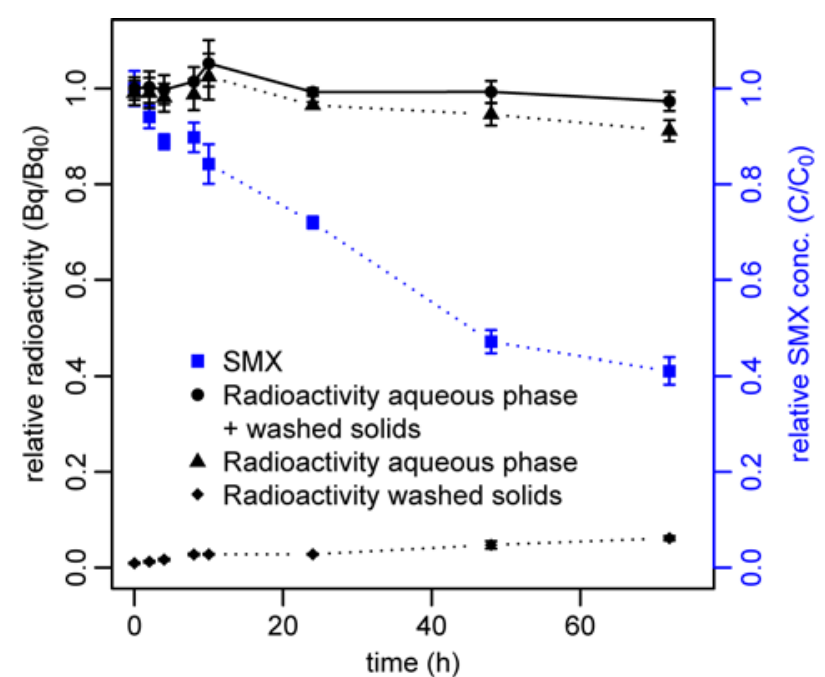

Figure 1. Biotransformation of ${ }^{14} \mathrm{C}-\mathrm{SMX}$. Radioactivity measured in washed solids, aqueous phase and the sum thereof as fractions of the total radioactivity measured after spiking. Relative concentration of SMX over time is shown in blue. Error bars represent the standard deviation from triplicate reactors.

\section{Identification of Biotransformation Products}

240 To first ensure that all analytes present in the supernatant samples of the ${ }^{14} \mathrm{C}$-labeled SMX biotransformation experiment (i.e., SMX and transformation products) could be fully recovered from chromatographic separation, separate radio-scintillating counting of the HPLC effluent was performed and revealed a mean recovery of $100.3 \pm 6.4 \%$ for the $0,24,28$, and 72 hour time points (relative to the total injected radioactivity of a single replicate). The HPLC-UV-DAD and the HPLC-LSC chromatogram both displayed a single dominant SMX peak in the first supernatant sample collected

246 from the biotransformation experiment (Figure 2). In the UV-DAD chromatogram, the intensity of the

247 SMX peak decreased with time, whereas a new peak emerged at a slightly higher retention time $(+0.72$

$248 \mathrm{~min}$ ). These observations are consistent with broadening and flattening of the SMX peak in the less

249 highly resolved HPLC-LSC chromatogram. The overall peak area in the HPLC-LSC chromatogram 250 (within the retention time window of 10-16 min as shown in Figure 2) showed only a slight decrease $251(-12.4 \pm 7.9 \%)$, suggesting that the major transformation products containing the ${ }^{14} \mathrm{C}$-labeled aniline 252 moiety elute at similar retention times as the parent compound. 


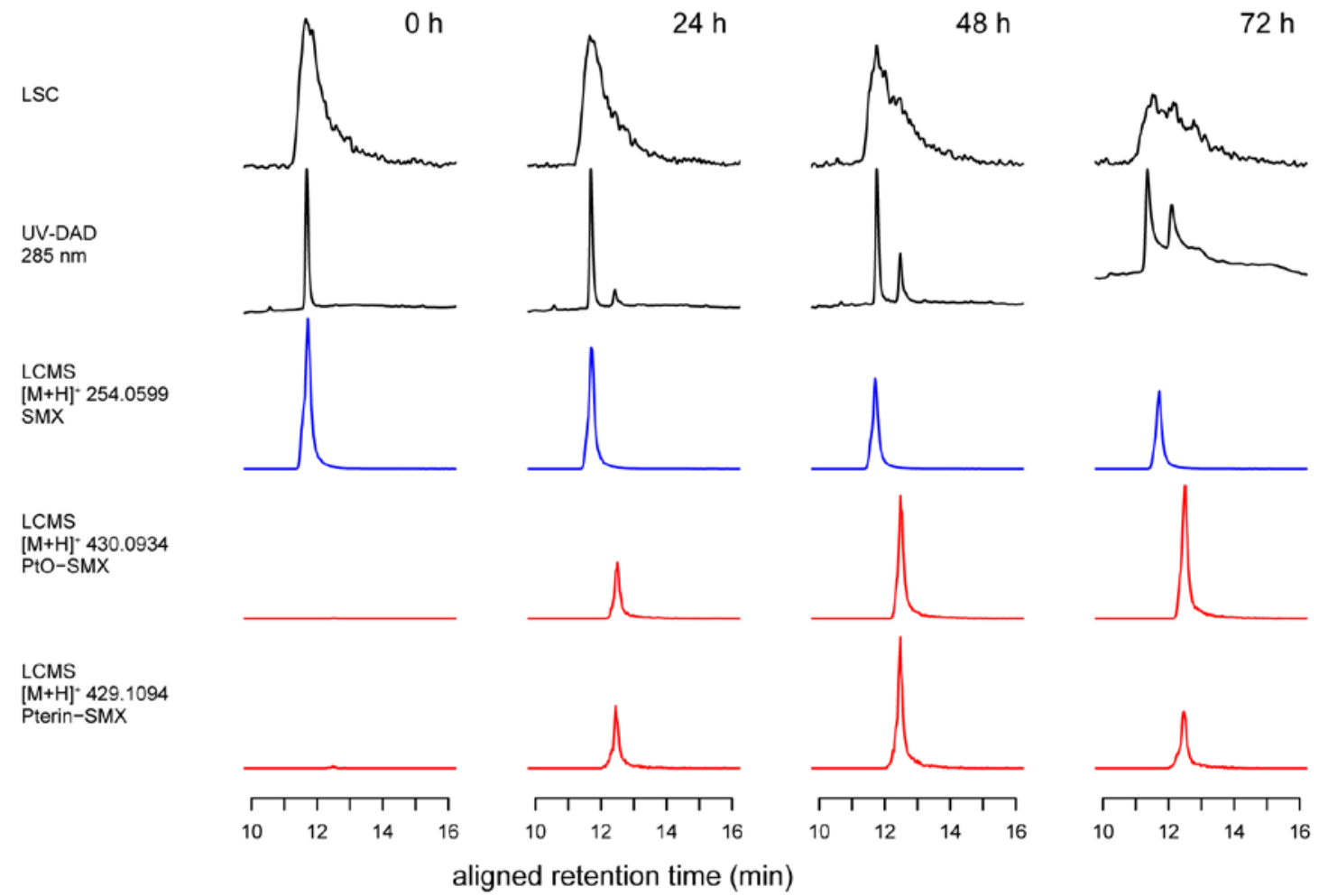

Figure 2. Chromatograms obtained from different detectors $0,24,48$ and 72 hours after start of the SMX biotransformation experiment. LC-MS and LSC signals were aligned with the UV-DAD signal using the peaks corresponding to SMX in the first sample $(0 \mathrm{~h})$. Signal intensities were normalized by maximal intensities over all four time points with the exception of the UVDAD signals for which normalization was performed for each sampling point separately (matrix interferences complicated comparisons of peak intensities and areas). Full chromatograms are shown in section S12 in the SI.

Suspect transformation product screening of samples from the batch experiments with non-labeled SMX led to the detection of 11 transformation products (TPs) (Table 1). In Table 1, the TPs are ordered according to their time-integrated intensities, which were calculated as the sum of measured peak areas at the different sampling time points. Interestingly, with PtO-SMX (a conjugate of 2,4(1H,3H)-pteridinedione, RT shift: $+0.79 \mathrm{~min})$, pterin-SMX (RT shift: $+0.77 \mathrm{~min})$ and Ac-OH-SMX (RT shift: $+0.72 \mathrm{~min}$ ), three of the five TPs with highest time-integrated intensities show similar retention time shifts relative to the parent as the emerging TP peak observed in the UV-DADchromatogram (Figure 2, section S12 in the SI). This finding suggests that the emerging TP peak in the UV-DAD-chromatogram is at least partially caused by the emergence of these three TPs. Moreover, with the exception of 3-amino-5-methylisoxazole (3A5MI), all detected TPs from SMX show similar RTs as SMX (RT shifts between -0.32 and +1.5 min, Table 1). Since all of these TPs 
still contain the radiolabeled aniline moiety, their emergence is consistent with the observation that the

272 overall peak area in the HPLC-LSC chromatogram, despite some broadening and flattening, is only

273 slightly reduced. This suggests that the majority of SMX might indeed be transformed to the TPs

274 given in Table 1 (for further discussion of mass balance aspects, see section on biotransformation

275 pathways).

Table 1. Summary of detected transformation products formed from the five investigated sulfonamides.

\begin{tabular}{|c|c|c|c|c|c|c|c|c|c|c|c|c|c|c|c|}
\hline TP & 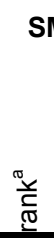 & 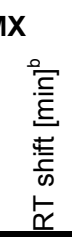 & 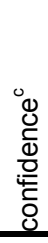 & 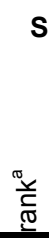 & 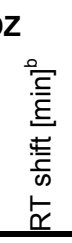 & $\begin{array}{l}0 \\
0 \\
0 \\
0 \\
0 \\
00 \\
0 \\
0 \\
0 \\
\end{array}$ & 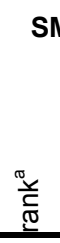 & 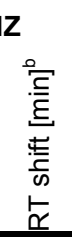 & $\begin{array}{l}0 \\
0 \\
0 \\
0 \\
0 \\
0 \\
0 \\
0 \\
0 \\
0 \\
0\end{array}$ & 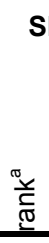 & 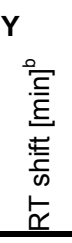 & 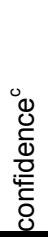 & 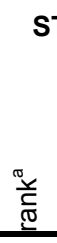 & 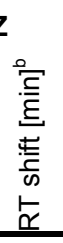 & $\begin{array}{l}0 \\
0 \\
0 \\
0 \\
0 \\
0 \\
0 \\
0 \\
0 \\
0 \\
\end{array}$ \\
\hline PtO-SA & 1 & 0.8 & $2 b$ & 1 & 1.8 & $2 b$ & 2 & 1.1 & $2 b$ & 4 & 1.5 & 3 & 1 & 1.5 & 3 \\
\hline pterin-SA & 2 & 0.8 & $2 b$ & 6 & 1.8 & $2 b$ & 5 & 1.0 & $2 b$ & 7 & 1.2 & 3 & 4 & 1.4 & 1 \\
\hline N4-formyl-SA & 3 & 1.0 & $2 b$ & 3 & 0.9 & $2 b$ & 4 & 0.1 & $2 b$ & 2 & 0.6 & $2 b$ & 3 & 1.1 & $2 b$ \\
\hline $\mathrm{Ac}-\mathrm{OH}-\mathrm{SA}$ & 5 & 0.8 & 3 & 2 & 1.3 & 3 & 1 & 1.1 & 3 & 1 & 1.3 & 3 & 2 & 1.2 & 3 \\
\hline$S A+O^{d}$ & 4 & -0.3 & 3 & 4 & -0.8 & 4 & 6 & -0.5 & 4 & 3 & -3.1 & 4 & & & \\
\hline pterin-SA + $\mathrm{H} 2 \mathrm{O}$ & 7 & 0.4 & 3 & 8 & 1.5 & 3 & & & & & & & & & \\
\hline N4-acetyl-SA & 8 & 1.5 & 1 & 5 & 1.8 & 1 & 3 & 0.9 & 1 & 5 & 1.3 & 1 & & & \\
\hline dihydropterin-SA & 9 & 0.6 & $2 b$ & & & & & & & & & & & & \\
\hline pterin-SA + O & 10 & 1.1 & 4 & 7 & 2.2 & 4 & & & & & & & & & \\
\hline $\mathrm{SA}+\mathrm{C} 3 \mathrm{H} 2 \mathrm{O} 3$ & 11 & 0.4 & 4 & & & & & & & 6 & 0.2 & 4 & & & \\
\hline $3 \mathrm{~A} 5 \mathrm{MI}$ & 6 & -4.1 & 1 & & & & & & & & & & & & \\
\hline $2 \mathrm{~A} 46 \mathrm{DP}^{\mathrm{e}}$ & & & & & & & 7 & -5.1 & $2 b$ & & & & & & \\
\hline
\end{tabular}

${ }^{a}$ For each parent SA, TPs are ranked according to time-integrated intensities across all samples. ${ }^{b} \mathrm{RT}$ shift indicates the difference in retention time between transformation product and parent SA. ${ }^{\circ}$ Confidence levels according to Schymanski et al. ${ }^{43}$ are assigned to all TPs (details in methods section and section $\mathrm{S} 7$ in the $\mathrm{SI}$ ). ${ }^{\mathrm{d}} \mathrm{For} \mathrm{SMX}+\mathrm{O}$, the molecular structure of N4hydroxy-SMX was proposed (details in section S7 in the SI). ${ }^{\text {e }}$-amino-4,6-dimethyl-pyrimidine.

HPLC-DAD-LSC chromatograms and recovered radioactive fractions from suspended solids and aqueous fractions of the SDZ biotransformation experiments confirmed the findings for SMX described above (section S9 in the SI). Similar to SMX, we observed a broadening and flattening of the sole LSC-peak present, and collection of the HPLC effluent and separate radio-scintillation counting again confirmed that all radioactivity could be recovered from the column (mean recovery for one replicate and time points $0,24,48,72 \mathrm{~h}: 104.3 \pm 3.9 \%$ ). Also, a decrease of the parent SDZ peak was observed in the UV-DAD chromatogram. All eight SDZ-TPs detected in the suspect screening (Table 1) corresponded to changes in molecular structure that were already observed for SMX. Furthermore, also for SMZ, SPY and STZ, similar product spectra as for SMX and SDZ were observed (Table 1). In particular, the TPs PtO-SA, Ac-OH-SA and formyl-SA were consistently 
observed to rank amongst the five TPs with the highest time-integrated intensities for all five sulfonamides studied.

Across all five sulfonamides, PtO-SA was the intensity-wise most dominant TP (i.e., it ranked first for three out of the five sulfonamides (SMX, SDZ and STZ) and had the lowest overall rank sum across all five sulfonamides). PtO-sulfonamides represent a modified pterin-sulfonamide and can presumably be formed by hydrolysis of the latter at position 2 of the pterin condensed ring structure (see discussion below). In our experiments, pterin-SAs were detected for all five investigated sulfonamides. The formation of pterin-SA via dihydropterin-SA has been described previously ${ }^{32,34,35}$ and is related to the actual mode of action of sulfonamides as antibacterial agents. Sulfonamides not only act as competitive inhibitors of dihydropteroate synthase but can also act as alternative substrates leading to the formation of pterin-sulfonamide conjugates for which retained antibacterial activity has recently been reported. ${ }^{38}$ Whereas this process has been described for pure cultures of bacteria and phytoplankton species, its potential significance in activated sludge has not been recognized so far.

\section{Biotransformation Pathways}

To obtain more information about the actual biotransformation pathways, we analyzed area-time trends of the detected TPs as presented in Figure 3 for SMX and in section S13 of the SI for SDZ, SPY, STZ and SMZ. For SMX, we observed that the TPs PtO-SMX, pterin-SMX and SMX+O, which we tentatively identified as N4-hydroxy-SMX (confidence level 3 according to Schymanksi et al., ${ }^{43}$ see Table 1 and details in section S7 in the SI), show a strong immediate increase upon incubation,

310 whereas N4-formyl-SMX was formed in larger amounts only later in the experiment. Similarly, the

311 N4-formyl TPs of the other sulfonamides also showed a pattern of delayed or slower increase (see

312 section S13), potentially indicating an indirect formation via other TPs. 


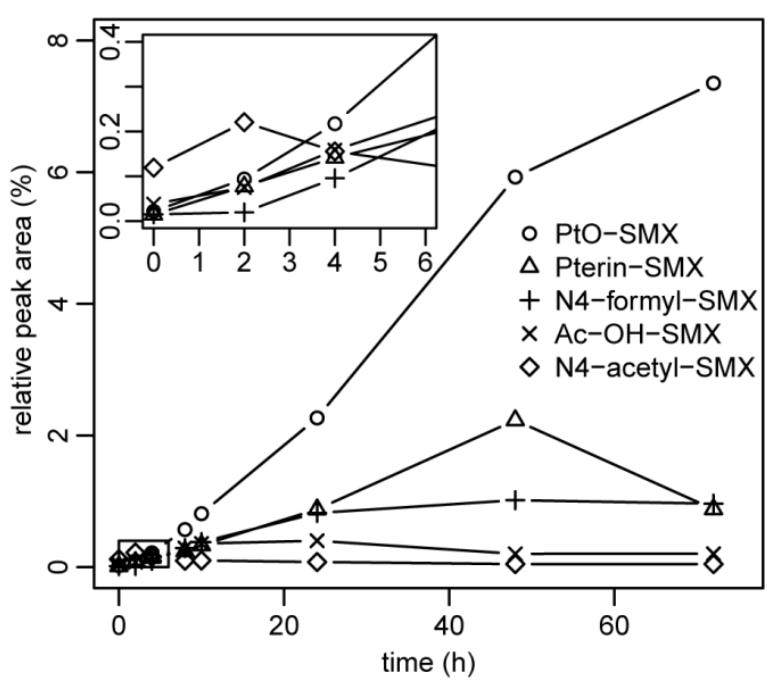

Figure 3. Time trends of transformation products of SMX. Peak areas of selected TPs are shown as percentage of the SMX peak area in the first sample after spiking. Area-time trends for the remaining TPs detected for SMX and TPS formed from the other investigated SAs are shown in section S13 in the SI.

To investigate the pterin-related biotransformation pathway further, we performed an experiment in which we directly spiked pterin-STZ to activated sludge. Compared to TP formation in STZ spike experiments, the observed peak areas of PtO-STZ, Ac-OH-STZ and formyl-STZ were clearly higher (in the case of PtO-STZ by more than one order of magnitude), which could not be explained by the minor amounts (approx. 5\%) of STZ parent compound present as impurity in the pterin-STZ standard (Figure S13.6 in the SI). This experiment revealed that not only PtO-STZ was formed from pterinSTZ, but also Ac-OH-STZ and formyl-STZ. Based on these results we developed a tentative, generalized pathway for the formation of PtO-SA, Ac-OH-SA and formyl-SA from pterin-SA as shown in Figure 4. The biotransformation of pterins to 2,4(1H,3H)-pteridinediones (here called PtOs) has earlier been observed in the context of folic acid biodegradation and can be catalyzed by the enzyme pterin deaminase. ${ }^{46,47}$ Although N4-formyl-SA and Ac-OH-SA TPs could theoretically also be formed directly from the parent sulfonamides, our results suggest that they are rather formed as part of the pterin-conjugate pathway, involving a series of hydrolysis, oxidation and decarboxylation reactions acting on either the pterin-SA or the PtO-SA as depicted in Figure 4. For SMX and SPY, a

331 TP with the mass of the possible intermediate $\mathrm{SA}+\mathrm{C}_{3} \mathrm{H}_{2} \mathrm{O}_{3}$ was additionally detected, and for SMX 332 and SDZ, a TP with the mass corresponding to pterin-SA $+\mathrm{H}_{2} \mathrm{O}$ was found, both lending additional support to the suggested pterin-conjugate pathway. Additionally, other potentially pterin-related TP 
peaks were detected corresponding to molecular formulas of pterin-SA+O. Detailed structural analyses of these TPs were complicated by the detected low peak areas and, hence, not further pursued. In addition to the pterin-conjugate pathway-related TPs, we also observed the previously described formation of N4-acetyl-SAs. ${ }^{37}$ Separate biotransformation experiments with a direct spike of acetylSMX confirmed rapid back-transformation to SMX, ${ }^{12}$ suggesting that SMX and acetyl-SMX are reversibly converted into each other (details in section S14 in the SI). Additionally, for SMX and and 2-amino-4,6-dimethyl-pyrimidine.

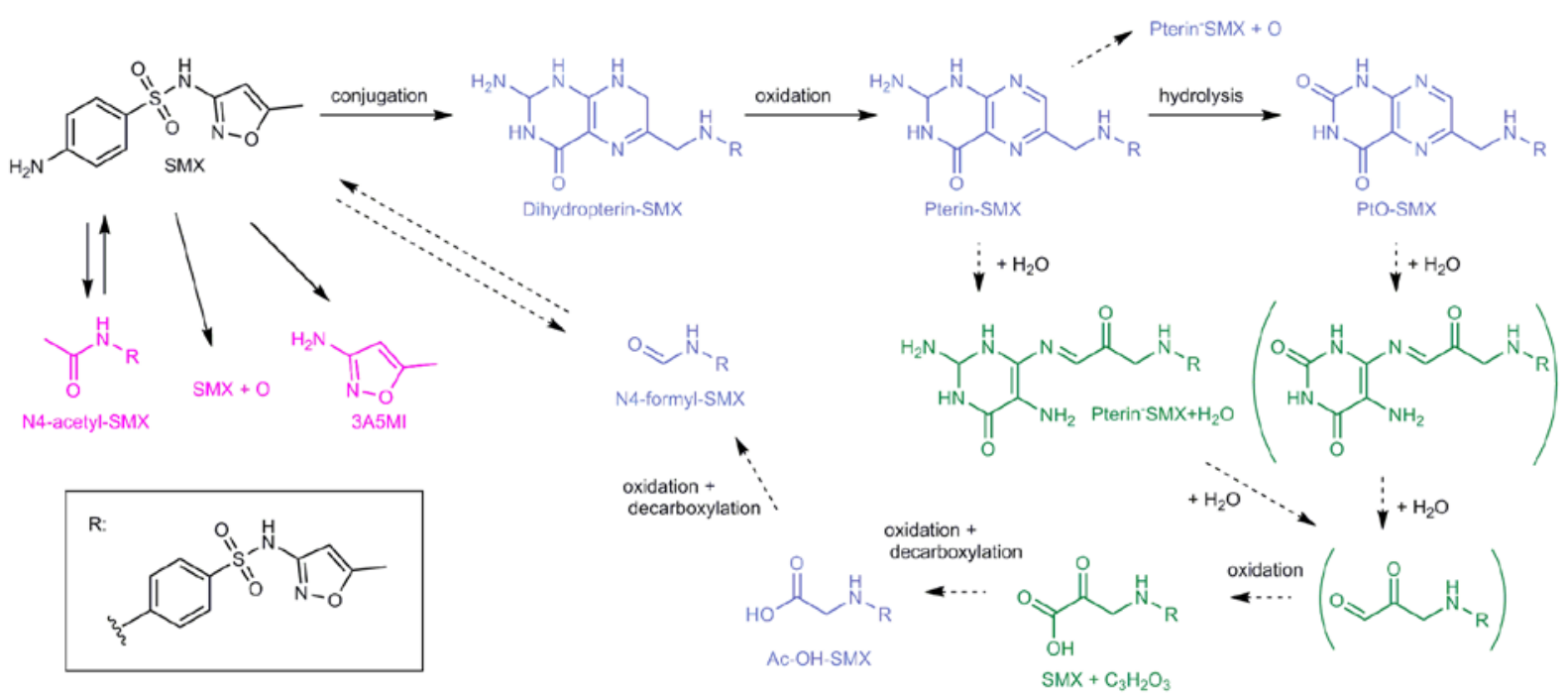

Figure 4. Proposed biotransformation pathway exemplified for SMX. As confirmed for STZ, PtO-SMX, Ac-OH-SMX and N4formyl-SMX are suggested to form via pterin-SMX and the detected intermediate dihydropterin-SMX (blue). TPs corresponding to masses of pterin-SMX+H2O and $\mathrm{SMX}+\mathrm{C} 3 \mathrm{H} 2 \mathrm{O} 3$ were detected but low peak areas did not allow for structure elucidation and the exact pathway to Ac-OH-SMX and N4-formyl-SMX remains speculative (green). N4-acetyl-SMX, 3A5MI and a TP with the mass SMX+O may form directly from SMX (pink).

We could quantify the concentrations of the N4-acetyl metabolites, the cleavage product 3A5MI and pterin-STZ based on mass spectrometric data. However, together they only accounted for less than five percent of the removed SMX at all time points. Lacking authentic standards, no accurate quantification was possible for the other TPs. To obtain an approximate assessment of the mass balance over the course of the experiment, we estimated relative ionization efficiencies for the remaining TPs (section S15 in SI) based on structural similarity to those TPs for which authentic standards were available. Based on authentic standards, the ionization efficiency of pterin-STZ was found to be one order of magnitude lower than that of STZ (i.e., ratio of instrument response factors of 
pterin-STZ over STZ: 0.1). Assuming that PtO-SAs exhibit similar ionization efficiencies as the structurally similar pterin-SAs, PtO-SA emerged as the TP that contributed most to the overall mass balance for all sulfonamides. Whereas the mass balance for SMX itself was closed when accounting for all observed TPs (Figure S15.1), some of the other sulfonamides, particularly SPY and STZ, revealed a lack of explainable mass loss, notably at later time points (Figures S15.2-5). This loss suggests that we might still lack a complete representation of later generation TPs formed out of the initial pterin-SA adducts. Although extrapolation of ionization efficiencies involves large uncertainties, ${ }^{48,49}$ and the calculated mass balances should therefore be interpreted with caution, they lead to strikingly similar conclusions as the results from the ${ }^{14} \mathrm{C}-\mathrm{SMX}$ experiments. In the UV-DAD chromatogram, one dominant TP peak was emerging with a similar retention time shift relative to SMX as the pterin-related TPs PtO-SMX, Ac-OH-SMX and pterin-SMX detected by LC-HRMS. Furthermore, the majority of the radioactivity eluted close to SMX and the above-mentioned TPs. DAD-UV chromatograms and the LSC-chromatograms thus support the dominance of the pterinconjugate pathway in the biotransformation of sulfonamides.

\section{Detection of Pterin-Sulfonamides in WWTPs Effluents}

By applying a suspect TP screening to effluent samples collected from nine Swiss WWTPs, the presence of pterin-conjugate pathway-related sulfonamide TPs could be confirmed. In Table 2, peak areas of TPs and effluent SMX are displayed as fractions of the influent SMX peak area (all peak areas were normalized using internal standards, quantified SMX influent concentrations were between 70 and $870 \mathrm{ng} / \mathrm{L}$ except for one WWTP where no detectable SMX concentrations were found). For seven out of the nine WWTPs, SMX peak areas showed a decline between $34 \%$ and $62 \%$ from influent to effluent. In one WWTP (WWTP5), an increase in SMX of 66\% between influent and effluent was observed. This can be explained by the large relative peak area of influent N4-acetyl-SMX, which is known to be transformed back to SMX in WWTPs. ${ }^{12}$ In the effluent samples, peaks corresponding to pterin-SMX or PtO-SMX were found for seven WWTPs, and in eight WWTP effluents peaks corresponding to Ac-OH-SMX and N4-formyl-SMX were detected. Only in two cases (Ac-OH-SMX in WWTP1 and PtO-SMX in WWTP6) higher or similar TP peak areas were found in influent samples compared to effluent samples. Reassuringly, we found no TP peaks in the effluent of the only WWTP 
in which no SMX or N4-acetyl-SMX was detected in the influent (WWTP3). Effluent TP peaks displayed peak areas that were roughly one to two orders of magnitude lower than the influent SMX peak areas (e.g., $\mathrm{PtO}_{\text {effluent }} / \mathrm{SMX}_{\text {influent: }} 13.7 \pm 8.5 \%$ ). However, because of the previously discussed rather low ionization efficiencies, relatively small pterin-SA peak areas can represent significant amounts of pterin-SAs. Therefore, the results presented in Table 2 do not only provide evidence for

390 the formation of pterin-SAs during activated sludge treatment in full-scale WWTPs, but show that the released pterin-conjugate pathway-related TPs potentially represent major fractions of the biotransformed SMX. Finally, the diversity of operational and design parameters of the nine WWTPs (see section S16 in SI for details) suggests that the formation of pterin-SAs is of general relevance and not limited to the activated sludge used in our biotransformation experiments.

Table 2. Transformation products from SMX detected in wastewater treatment plant effluents.

\section{Implications}

We demonstrate the significance of the biotransformation of sulfonamides through the pterinconjugate pathway and the formation of a suite of derivative transformation products in both batch biotransformation studies with activated sludge and in municipal wastewater treatment plants. Although sulfonamides have been previously reported to act as alternate substrates for DHPS and form pterin-SA conjugates, ${ }^{32}$ this is the first report of the dominant contribution of this transformation pathway during wastewater treatment.

The relevance of these findings is highlighted by a number of studies that have demonstrated the potential of diyhdropterin- and pterin-SAs to occupy the active site of the DHPS enzyme, ${ }^{32,38,50}$ and to exhibit antibacterial activity by inhibition of the DHPS enzyme. ${ }^{38}$ In a recent study, dihydropterin-STZ 
was even used as lead structure to develop novel antibacterial agents based on the replacement of dihydropterin with a quinoxaline moiety. ${ }^{51}$ In addition to sulfonamide conjugates, other transformation products of SMX modified at the N4-group, including N4-nitro-SMX and N4-hydroxy-SMX (here tentatively detected and denoted as SMX+O), have been shown to exhibit similar or even higher antibacterial activity than SMX. ${ }^{39}$ Although no potency information is currently available for the major transformation product $\mathrm{PtO}-\mathrm{SA}$, it is a pterin-related conjugate and may demonstrate similar antibacterial activities. Additionally, according to our results, only small fractions of sulfonamides were cleaved at the sulfonamide bridge resulting in transformation products with an undisputable loss of antibiotic activity. ${ }^{39}$ The fact that N4-acetyl-SMX was demonstrated here and by others ${ }^{12,52,53}$ to be readily back-transformed to parent SMX in different environments thus further raises the question of the potential of other pterin-conjugate pathway products such as PtO-SAs, N4-formyl-SA or Ac-OHSA to be transformed back to the parent sulfonamide in the environment. Taken together our results suggest that although activated sludge treatment in WWTPs reduces the load of parent sulfonamides to the environment, it may well lead to the formation and environmental release of sulfonamide transformation products with similar potential to exert antibiotic activity as the parent compounds.

More generally, our results emphasize the claim that quantification of removal of antibiotics alone is insufficient and that transformation products and pathways must be elucidated thoroughly to understand and evaluate the risks related with the usage and subsequent release of antibiotics to the environment. The same point has previously been underscored for other water treatment processes (e.g., aqueous ozonation ${ }^{54}$ ) and for other classes of biologically active micropollutants (e.g., steroid hormones, for which a variety of transformation reactions has been shown to lead to only minor structural modifications and hence transformation products with retained or even strongly enhanced endocrine-disrupting activities, ${ }^{26,55}$ or pesticide active ingredients ${ }^{27}$ ). Unfortunately, this point is still

432 often ignored in practice. Bioassays that allow measuring relevant endpoints such as antibiotic activity 433 in complex matrices and hence enable an effect-driven approach in transformation product analysis may support consideration of transformation products in future studies. ${ }^{56,57}$ 
Finally, our findings are also highly relevant in that they provide a potential explanation of seemingly contradictory findings on optimal conditions for sulfonamide removal in WWTPs. In a number of studies, an association of sulfonamide removal with the addition of readily available carbon sources or measures of heterotrophic activity was found. ${ }^{20-24,58}$ Yet, others provide evidence suggesting an

439 involvement of ammonia oxidizing microorganisms, ${ }^{25,59}$ such as correlation of SMX removal with 440 nitrifying activity in an enriched culture of ammonia oxidizing bacteria. ${ }^{25}$ In turn, this latter finding 441 stands in contradiction with the fact that sulfonamide degradation was mostly insensitive to inhibition 442 of the nitrifiers in batch experiments with activated sludge. ${ }^{45}$ Our results offer a new view on these discussions in that they demonstrate that sulfonamide biotransformation in activated sludge communities is apparently to a large extent related to their interference with folic acid synthesis. Because this pathway is integral to cellular production and maintenance, one can expect sulfonamide

446 biotransformation to pterin-SAs to correlate with bacterial growth. This in turn explains why both 447 nitrifier enrichment cultures and pure heterotrophic cultures both fed with their respective growth 448 substrates, i.e., ammonium or different carbon sources, respectively, show enhanced sulfonamide removal. Sulfonamide biotransformation has also been observed to occur readily under a number of conditions differing from those prevailing in aerated bioreactor experiments with activated sludge. For instance, sulfonamide removal has also been observed under anaerobic ${ }^{21,58,60}$ and anoxic ${ }^{21,61}$ conditions, in microbial communities from river sediments ${ }^{52}$ and river biofilms, ${ }^{8}$ and by different algal species. ${ }^{35}$ Based on our results, the observed ubiquitous trait of sulfonamide biotransformation in

454 microbial communities becomes a logical consequence of the transformation of sulfonamides through 455 the pterin-conjugate pathway. 


\section{Acknowledgements}

458 We thank Dr. Hans Peter E. Kohler, Dr. Adriano Joss, Dr. David R. Johnson und Dr. Michael A.

459 Stravs (Eawag) for fruitful discussions and Kevin Kroll (University of Applied Sciences and Arts

460 Northwestern Switzerland) for his help in the radioisotope laboratory. We thank the operators and the

461 staff of the WWTP ARA Neugut for providing activated sludge. We acknowledge financial support

462 from the European Research Council under the European Union's Seventh Framework Programme

463 (ERC grant agreement no. 614768, PROduCTS) and from the Swiss National Science Foundation

464 (grant agreement no. 310030L_160332). Dr. Anze Zupanic (Eawag) and Dr. Richard E. Lee (St. Jude

465 Children's Research Hospital, Memphis, USA) are acknowledged for helpful comments on the

466 manuscript.

468 Supporting Information

469 Information on chemical reference compounds; details on biotransformation experiments; analytical

470 details; listing of parameters used for the suspect transformation product screening; structure

471 elucidation of transformation products by analysis of $\mathrm{MS}^{2}$ fragmentation spectra; mass spectra;

472 additional results of biotransformation and control experiments and analysis of WWTP samples;

473 estimation of mass balances. 


\section{References}

477 1. Kümmerer, K., Antibiotics in the aquatic environment--a review--part I. Chemosphere 2009,

2. Michael, I.; Rizzo, L.; McArdell, C. S.; Manaia, C. M.; Merlin, C.; Schwartz, T.; Dagot, C.; FattaKassinos, D., Urban wastewater treatment plants as hotspots for the release of antibiotics in the environment: A review. Water Res. 2013, 47, 957-995.

3. Kümmerer, K., Antibiotics in the aquatic environment--a review--part II. Chemosphere 2009, 75, 435-441.

4. de Voogt, P.; Janex-Habibi, M. L.; Sacher, F.; Puijker, L.; Mons, M., Development of a common priority list of pharmaceuticals relevant for the water cycle. Water Sci. Technol. 2009, 59, 3946.

5. Grung, M.; Kallqvist, T.; Sakshaug, S.; Skurtveit, S.; Thomas, K. V., Environmental assessment of Norwegian priority pharmaceuticals based on the EMEA guideline. Ecotoxicol. Environ. Saf. 2008, 71, 328-340.

6. Johnson, A. C.; Keller, V.; Dumont, E.; Sumpter, J. P., Assessing the concentrations and risks of toxicity from the antibiotics ciprofloxacin, sulfamethoxazole, trimethoprim and erythromycin in European rivers. Sci. Total Environ. 2015, 511, 747-755.

7. Hruska, K.; Franek, M., Sulfonamides in the environment: a review and a case report. Vet. Med. Czech. 2012, 57, 1-35.

8. Vila-Costa, M.; Gioia, R.; Acena, J.; Perez, S.; Casamayor, E. O.; Dachs, J., Degradation of sulfonamides as a microbial resistance mechanism. Water Res. 2017, 115, 309-317.

9. Straub, J. O., Aquatic Environmental Risk Assessment for Human Use of the Old Antibiotic Sulfamethoxazole in Europe. Environ. Toxicol. Chem. 2016, 35, 767-779.

10. Stoob, K.; Singer, H. P.; Mueller, S. R.; Schwarzenbach, R. P.; Stamm, C. H., Dissipation and transport of veterinary sulfonamide antibiotics after manure application to grassland in a small catchment. Environ. Sci. Technol. 2007, 41, 7349-7355.

11. Batt, A. L.; Snow, D. D.; Aga, D. S., Occurrence of sulfonamide antimicrobials in private water wells in Washington County, Idaho, USA. Chemosphere 2006, 64, 1963-1971.

12. Göbel, A.; Thomsen, A.; McArdell, C. S.; Joss, A.; Giger, W., Occurrence and sorption behavior of sulfonamides, macrolides, and trimethoprim in activated sludge treatment. Environ. Sci. Technol. 2005, 39, 3981-3989.

13. Johnson, A. C.; Jürgens, M. D.; Nakada, N.; Hanamoto, S.; Singer, A. C.; Tanaka, H., Linking changes in antibiotic effluent concentrations to flow, removal and consumption in four different UK sewage treatment plants over four years. Environ. Pollut. 2017, 220, 919-926.

14. Xu, W. H.; Zhang, G.; Li, X. D.; Zou, S. C.; Li, P.; Hu, Z. H.; Li, J., Occurrence and elimination of antibiotics at four sewage treatment plants in the Pearl River Delta (PRD), South China. Water Res. 2007, 41, 4526-4534.

15. Carballa, M.; Omil, F.; Lema, J. M.; Llompart, M.; Garcia-Jares, C.; Rodriguez, I.; Gomez, M.; Ternes, T., Behavior of pharmaceuticals, cosmetics and hormones in a sewage treatment plant. Water Res. 2004, 38, 2918-2926.

16. Singer, A. C.; Järhult, J. D.; Grabic, R.; Khan, G. A.; Lindberg, R. H.; Fedorova, G.; Fick, J.; Bowes, M. J.; Olsen, B.; Soderstrom, H., Intra- and inter-pandemic variations of antiviral, antibiotics and decongestants in wastewater treatment plants and receiving rivers. PLoS One 2014, 9, e108621.

17. Peng, X.; Wang, Z.; Kuang, W.; Tan, J.; Li, K., A preliminary study on the occurrence and behavior of sulfonamides, ofloxacin and chloramphenicol antimicrobials in wastewaters of two sewage treatment plants in Guangzhou, China. Sci. Total Environ. 2006, 371, 314-322.

18. Ternes, T.; Joss, A., Human pharmaceuticals, hormones and fragrances. IWA publishing: 2007.

19. Onesios, K. M.; Jim, T. Y.; Bouwer, E. J., Biodegradation and removal of pharmaceuticals and personal care products in treatment systems: a review. Biodegradation 2009, 20, 441-466. 
20. Majewsky, M.; Gallé, T.; Yargeau, V.; Fischer, K., Active heterotrophic biomass and sludge retention time (SRT) as determining factors for biodegradation kinetics of pharmaceuticals in activated sludge. Bioresour. Technol. 2011, 102, 7415-7421.

21. Alvarino, T.; Nastold, P.; Suarez, S.; Omil, F.; Corvini, P. F.; Bouju, H., Role of biotransformation, sorption and mineralization of ${ }^{14} \mathrm{C}$-labelled sulfamethoxazole under different redox conditions. Sci. Total Environ. 2016, 542, 706-715.

22. Gauthier, H.; Yargeau, V.; Cooper, D. G., Biodegradation of pharmaceuticals by Rhodococcus rhodochrous and Aspergillus niger by co-metabolism. Sci. Total Environ. 2010, 408, 17011706.

23. Müller, E.; Schüssler, W.; Horn, H.; Lemmer, H., Aerobic biodegradation of the sulfonamide antibiotic sulfamethoxazole by activated sludge applied as co-substrate and sole carbon and nitrogen source. Chemosphere 2013, 92, 969-978.

24. Fernandez-Fontaina, E.; Gomes, I. B.; Aga, D. S.; Omil, F.; Lema, J. M.; Carballa, M., Biotransformation of pharmaceuticals under nitrification, nitratation and heterotrophic conditions. Sci. Total Environ. 2016, 541, 1439-1447.

25. Kassotaki, E.; Buttiglieri, G.; Ferrando-Climent, L.; Rodriguez-Roda, I.; Pijuan, M., Enhanced sulfamethoxazole degradation through ammonia oxidizing bacteria co-metabolism and fate of transformation products. Water Res. 2016, 94, 111-119.

26. Cwiertny, D. M.; Snyder, S. A.; Schlenk, D.; Kolodziej, E. P., Environmental Designer Drugs: When Transformation May Not Eliminate Risk. Environ. Sci. Technol. 2014, 48, 11737-11745.

27. Fenner, K.; Canonica, S.; Wackett, L. P.; Elsner, M., Evaluating pesticide degradation in the environment: blind spots and emerging opportunities. Science 2013, 341, 752-758.

28. Reis, P. J.; Reis, A. C.; Ricken, B.; Kolvenbach, B. A.; Manaia, C. M.; Corvini, P. F.; Nunes, O. C., Biodegradation of sulfamethoxazole and other sulfonamides by Achromobacter denitrificans PR1. J. Hazard. Mater. 2014, 280, 741-749.

29. Jiang, B.; Li, A.; Cui, D.; Cai, R.; Ma, F.; Wang, Y., Biodegradation and metabolic pathway of sulfamethoxazole by Pseudomonas psychrophila HA-4, a newly isolated cold-adapted sulfamethoxazole-degrading bacterium. Appl Microbiol Biotechnol 2014, 98, 4671-4681.

30. Deng, Y.; Mao, Y.; Li, B.; Yang, C.; Zhang, T., Aerobic Degradation of Sulfadiazine by Arthrobacter spp.: Kinetics, Pathways, and Genomic Characterization. Environ. Sci. Technol. 2016, 50, 9566-9575.

31. Bouju, H.; Ricken, B.; Beffa, T.; Corvini, P. F.; Kolvenbach, B. A., Isolation of bacterial strains capable of sulfamethoxazole mineralization from an acclimated membrane bioreactor. Appl Environ Microbiol 2012, 78, 277-279.

32. Roland, S.; Ferone, R.; Harvey, R. J.; Styles, V. L.; Morrison, R. W., The characteristics and significance of sulfonamides as substrates for Escherichia coli dihydropteroate synthase. $J$. Biol. Chem. 1979, 254, 10337-10345.

33. Bock, L.; Miller, G. H.; Schaper, K. J.; Seydel, J. K., Sulfonamide structure-activity relationships in a cell-free system. 2. Proof for the formation of a sulfonamide-containing folate analog. J. Med. Chem. 1974, 17, 23-28.

34. Richter, M. K.; Focks, A.; Siegfried, B.; Rentsch, D.; Krauss, M.; Schwarzenbach, R. P.; Hollender, J., Identification and dynamic modeling of biomarkers for bacterial uptake and effect of sulfonamide antimicrobials. Environ. Pollut. 2013, 172, 208-215.

35. Stravs, M. A.; Pomati, F.; Hollender, J., Exploring micropollutant biotransformation in three freshwater phytoplankton species. Environ. Sci. Proc. Imp. 2017, 19, 822-832.

36. Larcher, S.; Yargeau, V., Biodegradation of sulfamethoxazole by individual and mixed bacteria. Appl. Microbiol. Biotechnol. 2011, 91, 211-218.

37. Majewsky, M.; Glauner, T.; Horn, H., Systematic suspect screening and identification of sulfonamide antibiotic transformation products in the aquatic environment. Anal. Bioanal. Chem. 2015, 407, 5707-5717. 
38. Zhao, Y.; Shadrick, W. R.; Wallace, M. J.; Wu, Y. N.; Griffith, E. C.; Qi, J. J.; Yun, M. K.; White, S. W.; Lee, R. E., Pterin-sulfa conjugates as dihydropteroate synthase inhibitors and antibacterial agents. Bioorg. Med. Chem. Lett. 2016, 26, 3950-3954.

39. Majewsky, M.; Wagner, D.; Delay, M.; Bräse, S.; Yargeau, V.; Horn, H., Antibacterial activity of sulfamethoxazole transformation products (TPs): general relevance for sulfonamide TPs modified at the para position. Chem. Res. Toxicol. 2014, 27, 1821-1828.

40. Gulde, R.; Meier, U.; Schymanski, E. L.; Kohler, H. P.; Helbling, D. E.; Derrer, S.; Rentsch, D.; Fenner, K., Systematic Exploration of Biotransformation Reactions of Amine-Containing Micropollutants in Activated Sludge. Environ. Sci. Technol. 2016, 50, 2908-2920.

41. Abellan, M. N.; Gimenez, J.; Esplugas, S., Photocatalytic degradation of antibiotics: The case of sulfamethoxazole and trimethoprim. Catal. Today 2009, 144, 131-136.

42. Espinosa-Mansilla, A.; Salinas, F.; De Orbe Paya, I., Simultaneous Determination of Sulfadiazine, Doxycycline, Furaltadone and Trimethoprim by Partial Least-Squares Multivariate Calibration. Anal. Chim. Acta 1995, 313, 103-112.

43. Schymanski, E. L.; Jeon, J.; Gulde, R.; Fenner, K.; Ruff, M.; Singer, H. P.; Hollender, J., Identifying small molecules via high resolution mass spectrometry: communicating confidence. Environ. Sci. Technol. 2014, 48, 2097-2098.

44. Moschet, C.; Piazzoli, A.; Singer, H.; Hollender, J., Alleviating the Reference Standard Dilemma Using a Systematic Exact Mass Suspect Screening Approach with Liquid Chromatography-High Resolution Mass Spectrometry. Anal. Chem. 2013, 85, 10312-10320.

45. Men, Y.; Achermann, S.; Helbling, D. E.; Johnson, D. R.; Fenner, K., Relative contribution of ammonia oxidizing bacteria and other members of nitrifying activated sludge communities to micropollutant biotransformation. Water Res. 2016, 109, 217-226.

46. Rappold, H.; Bacher, A., Bacterial degradation of folic acid. J. Gen. Microbiol. 1974, 85, 283290.

47. Jayaraman, A.; Thandeeswaran, M.; Priyadarsini, U.; Sabarathinam, S.; Nawaz, K. A.; Palaniswamy, M., Characterization of unexplored amidohydrolase enzyme-pterin deaminase. Appl. Microbiol. Biotechnol. 2016, 100, 4779-4789.

48. Oss, M.; Kruve, A.; Herodes, K.; Leito, I., Electrospray ionization efficiency scale of organic compounds. Anal. Chem. 2010, 82, 2865-2872.

49. Cech, N. B.; Enke, C. G., Practical implications of some recent studies in electrospray ionization fundamentals. Mass Spectrom. Rev. 2001, 20, 362-387.

50. Yun, M. K.; Wu, Y.; Li, Z.; Zhao, Y.; Waddell, M. B.; Ferreira, A. M.; Lee, R. E.; Bashford, D.; White, S. W., Catalysis and sulfa drug resistance in dihydropteroate synthase. Science 2012, 335, 1110-1114.

51. El-Attar, M. A. Z.; Elbayaa, R. Y.; Shaaban, O. G.; Habib, N. S.; Abdel Wahab, A. E.; Abdelwahab, I. A.; El-Hawash, S. A. M., Design, synthesis, antibacterial evaluation and molecular docking studies of some new quinoxaline derivatives targeting dihyropteroate synthase enzyme. Bioorg. Chem. 2017, 76, 437-448.

52. Radke, M.; Lauwigi, C.; Heinkele, G.; Mürdter, T. E.; Letzel, M., Fate of the antibiotic sulfamethoxazole and its two major human metabolites in a water sediment test. Environ. Sci. Technol. 2009, 43, 3135-3141.

53. Förster, M.; Laabs, V.; Lamshoft, M.; Groeneweg, J.; Zühlke, S.; Spiteller, M.; Krauss, M.; Kaupenjohann, M.; Amelung, W., Sequestration of manure-applied sulfadiazine residues in soils. Environ. Sci. Technol. 2009, 43, 1824-1830.

54. Dodd, M. C.; Rentsch, D.; Singer, H. P.; Kohler, H. P.; von Gunten, U., Transformation of betalactam antibacterial agents during aqueous ozonation: reaction pathways and quantitative bioassay of biologically-active oxidation products. Environ. Sci. Technol. 2010, 44, 5940-5948. 55. Qu, S.; Kolodziej, E. P.; Long, S. A.; Gloer, J. B.; Patterson, E. V.; Baltrusaitis, J.; Jones, G. D.; Benchetler, P. V.; Cole, E. A.; Kimbrough, K. C.; Tarnoff, M. D.; Cwiertny, D. M., Product-toparent reversion of trenbolone: unrecognized risks for endocrine disruption. Science 2013, 342, 347-351. 
56. Jahnke, A.; Mayer, P.; Schäfer, S.; Witt, G.; Haase, N.; Escher, B. I., Strategies for Transferring Mixtures of Organic Contaminants from Aquatic Environments into Bioassays. Environ. Sci. Technol. 2016, 50, 5424-5431.

57. Escher, B. I.; Fenner, K., Recent advances in environmental risk assessment of transformation products. Environ. Sci. Technol. 2011, 45, 3835-3847.

58. Fan, C. H.; Yang, C. W.; Chang, B. V., Anaerobic degradation of sulfamethoxazole by mixed cultures from swine and sewage sludge. Environ. Technol. 2017, 10.1080/09593330.2017.1384510.

59. Torresi, E.; Fowler, S. J.; Polesel, F.; Bester, K.; Andersen, H. R.; Smets, B. F.; Plosz, B. G.; Christensson, M., Biofilm Thickness Influences Biodiversity in Nitrifying MBBRs-Implications on Micropollutant Removal. Environ. Sci. Technol. 2016, 50, 9279-9288.

60. Gonzalez-Gil, L.; Mauricio-Iglesias, M.; Serrano, D.; Lema, J. M.; Carballa, M., Role of methanogenesis on the biotransformation of organic micropollutants during anaerobic digestion. Sci. Total Environ. 2017, 622-623, 459-466.

61. Hai, F. I.; Li, X.; Price, W. E.; Nghiem, L. D., Removal of carbamazepine and sulfamethoxazole by MBR under anoxic and aerobic conditions. Bioresour. Technol. 2011, 102, 10386-10390. 